\title{
Proposed Synopsis Frontal Signal Actuated Speech Synthesizer Through Fnir
}

\author{
S Srilekha* \\ Information and communication Engineering, India
}

Received: 眥: November 27, 2018; Published: 制: December 06, 2018

*Corresponding author: S Srilekha, Information and communication Engineering, Chennai, India

\begin{abstract}
Self-movement is a vital role to lead a peaceful life for normal human beings. The concept of this paper is to help the person who suffers from various impairment physically such as deaf-dumb, unable to speak, etc. they need person to support for their regular activities. This paper mainly focuses on the mind power via FNIR with the oxygenated blood flow in brain. The system deals on speech synthesizer with the knowledge of embedded controllers and audio pre-recorder which shows a dynamic part in day to day communication. First, the acquired signal is processed for feature extraction and classification to analyze the discrepancies in speech of patients. The main advantages of this system are to prompt their necessities to manage their life as a typical individual.
\end{abstract}

\section{Introduction}

Electroencephalography (EEG) is an electrophysiological monitoring method to record electrical activity of the brain. It is typically noninvasive, with the electrodes placed along the scalp, although invasive electrodes are sometimes used in specific applications. EEG measures voltage fluctuations resulting from ionic current within the neurons of the brain. In clinical contexts, EEG refers to the recording of the brain's spontaneous electrical activity over a period, as recorded from multiple electrodes placed on the scalp. Diagnostic applications generally focus on the spectral content of EEG, that is, the type of neural oscillations (popularly called "brain waves") that can be observed in EEG signals. The brain's electrical charge is maintained by billions of neurons. Neurons are electrically charged (or "polarized") by membrane transport proteins that pump ions across their membranes. Neurons are constantly exchanging ions with the extracellular milieu, for example to maintain resting potential and to propagate action potentials. Ions of similar charge repel each other and when many ions are pushed out of many neurons at the same time, they can push their neighbors, who push their neighbors, and so on, in a wave. This process is known as volume conduction. When the wave of ions reaches the electrodes on the scalp, they can push or pull electrons on the metal in the electrodes. Since metal conducts the push and pull of electrons easily, the difference in push or pull voltages between any two electrodes can be measured by a voltmeter. Recording these voltages over time gives us the EEG.
In conventional scalp EEG, the recording is obtained by placing electrodes on the scalp with a conductive gel or paste, usually after preparing the scalp area by light abrasion to reduce impedance due to dead skin cells. Many systems typically use electrodes, each of which is attached to an individual wire. Some systems use caps or nets into which electrodes are embedded; This is particularly common when high-density arrays of electrodes are needed. Electrode locations and names are specified by the International 10-20 system for most clinical and research applications (except when high-density arrays are used). This system ensures that the naming of electrodes is consistent across laboratories. In most clinical applications, 19 recording electrodes (plus ground and system reference) are used. A smaller number of electrodes are typically used when recording EEG from neonates. Additional electrodes can be added to the standard set-up when a clinical or research application demands increased spatial resolution for a particular area of the brain. High-density arrays (typically via cap or net) can contain up to 256 electrodes more-or-less evenly spaced around the scalp.

Each electrode is connected to one input of a differential amplifier (one amplifier per pair of electrodes); A common system reference electrode is connected to the other input of each differential amplifier. These amplifiers amplify the voltage between the active electrode and the reference (typically 1,000-100,000 
times, or $60-100 \mathrm{~dB}$ of voltage gain). In analog EEG, the signal is then filtered (next paragraph) and the EEG signal is output as the deflection of pens as paper passes underneath. Most EEG systems these days, however, are digital, and the amplified signal is digitized via an analog-to-digital converter, after being passed through an anti-aliasing filter. Analog-to-digital sampling typically occurs at 256-512 Hz in clinical scalp EEG; sampling rates of up to $20 \mathrm{kHz}$ are used in some research applications. Figure 1 shows the EEG Waveforms for different activities of the human beings.

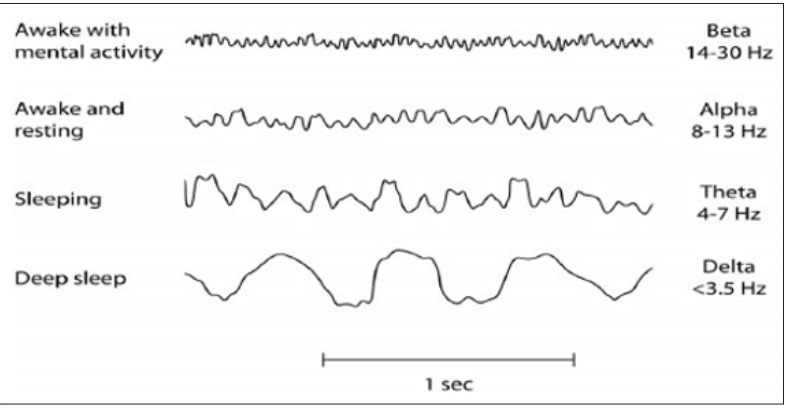

Figure 1: EEG Waveforms.

\section{Related Works}

The works that are closely related to ours are, Sim Kok Swee et al. [1], have proposed the methods for using the Emotiv EPOC headset, two methods are employed to process the EEG signal data into mental commands. The first method is the EmoEngine from the Emotiv. Another method is known as Fast Fourier Analysis and EEG Classification. Nikhil Shinde et al., have proposed the methods for utilizing EEG and EOG to operate the wheelchair [2-6]. The design incorporates a safety controller with peripheral safety sensors that override the user command and stop the wheelchair when it detects an obstacle in its path. Kazuo Kiguchi et al. [7], have proposed the user's motion estimation method was proposed to control the wearable robots based on the user's motion intention. EEG signals are applied to compensate for the lack of the EMG signals. M Akila et al., have proposed to analyze the brain wave signals, it uses only single electrode headset based on EEG sensors which will monitor the eye blinks, attention mode, and meditation mode, but not going to monitor the rays coming from the scalp. Nikhil R Folane et al., have proposed the gold-plated dry electrodes were used for system which consist a single channel having three contacts points i.e. EEG, Reference and Ground. Reshmi G et al., have proposed in this work for designing a BCI system five class motor imagery EEG is used. EEG recorded from the sensory motor cortex is analyzed using wavelet transform. Then classified using Support Vector Machine. Rifai Chai et al., it uses only two EEG channels and a better feature extractor to improve the portability and accuracy. In addition, two different features extraction methods, PSD and HHT energy are compared to improve accuracy using a GA [8].

\section{Proposed System}

The Proposed system is mainly depending upon EEG waveforms. Brainwave sensor, Mat lab and Wheelchair Controller are the key parameters of system. Figure 2 illustrates conceptual diagram of the proposed system. The block diagram consists of the following modules:

a) EEE Acquisition Unit

b) Bluetooth Transmitter

c) Bluetooth Receiver

d) Software Platform

i. Feature Extraction

ii. Feature Classification

e) Audio Controller

i. $\quad$ ARDUINO Microcontroller

ii. $\quad$ RF Module

iii. Activation of pre-recorder

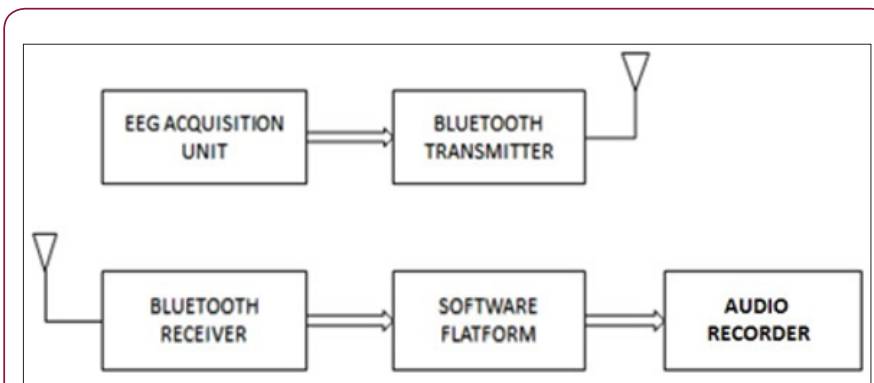

Figure 2: Block Diagram Representation.

\section{EEG Acquisition Unit}

FNIR systems provide real-time monitoring of tissue oxygenation in the brain as subjects take tests, perform tasks, or receive stimulation and allows researchers to quantitatively assess brain functions such as attention, memory, planning and problem solving while individuals perform cognitive tasks. It eliminates many of the drawbacks of MRI and provides a safe, affordable, noninvasive solution for cognitive function assessment. The FNIR device provides relative change in hemoglobin levels, calculated using a modified Beer- Lambert law. The powerful spectroscopy imaging tool measures NIR light absorbance in blood of hemoglobin with and without oxygen and provides about ongoing brain activity like functional MRI studies (Figure 3).

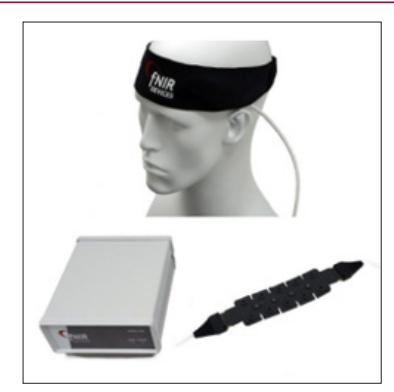

Figure 3: BIOPAC FNIR Sensor.

\section{Signal Processing}

The processing of the different signals is done in two phases namely Feature Extraction and Feature Classification. 
Feature Extraction: In this paper, the processing of EEG data is done using the new technique, Fast Fourier Transform Analysis and Wavelet transform for feature extraction. First, the raw EEG data is retrieved. This EEG data had not been processed in the way as EmoState. Therefore, Fast Fourier Transform is applied to convert the complicated EEG waveforms into a simpler waveform. This processing method can produce a waveform which is easier for analyses and differentiate from others. Training for the mental command is also required for this approach. The waveforms after the FFT process are recorded during the training session. Then, the detection of the mental command is carried out by classifying the detected waveform to the recorded waveform.

Feature Classification: Support Vector Machine (SVM) is primarily a classifier method that performs classification tasks by constructing hyper planes in a multidimensional space that separates cases of different class labels. The SVM must be trained, just as an Artificial Neural Network must be trained. It maps training data in the input space into a high dimensional feature space. It determines a linear decision boundary in the feature space by constructing the optimal separating hyper plane distinguishing the classes. This allows the SVM to achieve a nonlinear boundary in the input space. The support vectors are those points in the input space which best define the boundary between the classes. Potentially difficult computations in the feature space are avoided by using a kernel function, which allows computations to be performed in the input space. Figure 4 demonstrates the System architecture in which the feature classification from the data acquisition unit and then the output is sent to the control unit.

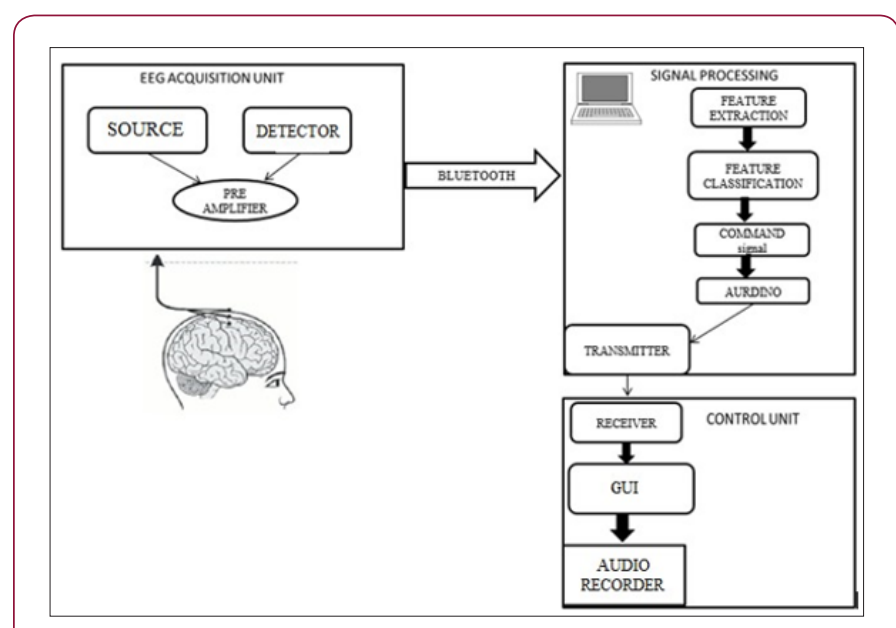

Figure 4: System Architecture.

\section{AUDIO Controller}

The Audio is controlled by programming in ARDUINO Microcontroller, then transmitting and receiving the signals/values through the RF module. The following is the operations of these three modules.
ARDUINO Microcontroller: The controller board for the controlling the entire electrical wheelchair is Arduino Uno microcontroller board. The Arduino Uno microcontroller is selected due to it consists of enough I/O pins. It can control the entire electrical wheelchair system. Besides, Arduino Uno microcontroller is relatively small size.

4RF Module: This RF module comprises of an RF Transmitter and an RF Receiver. The transmitter/receiver (Tx/Rx) pair operates at a frequency of $434 \mathrm{MHz}$ an $\mathrm{RF}$ transmitter receives serial data and transmits it wirelessly through RF through its antenna connected at pin4. The transmission occurs at the rate of $1 \mathrm{Kbps}-10 \mathrm{Kbps}$. The transmitted data is received by an $\mathrm{RF}$ receiver operating at the same frequency as that of the transmitter.

Audio Recorder: RF receiver receives the signal from arduino and sends it to GUI to operate the voice module. Four commands are primarily tested for accuracy and efficiency. Voice module has some recorded audio speech for testing the above commands.

\section{Conclusion}

Cognitive tasks captured will be used for computer interface. Main goal of the proposed work to convert mental task related to text into speech. Here input to the proposed system will be brain signals, output will be analog speech signals which are audible through speakers. This is helpful to people especially who cannot walk and speak.

\section{References}

1. Sim Kok Swee, Lim Zheng You (2016) Fast Fourier Analysis and EEG Classification Brainwave Controlled Wheelchair. IEEE.

2. Brice R, Cuntai G, Haihong Z, C Wang, C Teo, et al. (2010) A Brain wheelchair to navigate in familiar environment. IEEE Trans Neural Syst Rehabil Eng 18(6): 590-598.

3. I Iturratte, JM Antelis, A Kubler, J Minguez (2014) A noninvasive brainactuated wheelchair based on a P300 neurophysiological protocol and automated navigation. IEEE Trans Robot 25(3): 278-288.

4. Vaibhav G, G Prasad, D Coyle, L Behera, TM McGinnity (2014) EEG-Based Mobile Robot Control through an Adaptive Brain- Robot Interface. IEEE transaction on systems, man, and cybernetics: Systems 44(9): 12781285.

5. Luzheng Bi, Xin An Fan, Yili Liu (2013) EEG-Based Brain-Controlled Mobile Robots: A Survey. IEEE Transactions on Human-Machine Systems 43(2): 161-176.

6. Wolpaw JR, Birbaumer N, McFarland DJ, Pfurtscheller G, Vaughan TM (2002) Brain-computer interfaces for communication and control. Clin Neurophysiol 113(6): 767-791.

7. K Kiguchi, Y Hayashi (2012) An EMG-Based Control for an UpperLimb Power-Assist Exoskeleton Robot. IEEE Trans Systems, Man and Cybernetics 42(4): 1064-1071.

8. Jenita Amali Rani, A Umamakeswari (2015) Electroencephalogrambased Brain Controlled Robotic Wheelchair. Indian Journal of Sci and Tech 8(S9): 188-197. 


\section{ISSN: 2574-1241}

DOI: 10.26717/BJSTR.2018.11.002154

S Srilekha. Biomed J Sci \& Tech Res

(c) (i) This work is licensed under Creative

BY Commons Attribution 4.0 License

Submission Link: https://biomedres.us/submit-manuscript.php

$\begin{array}{ll}\text { BIOMEDICAL } & \text { Assets of Publishing with us } \\ \text { RESEARCHES } & \text { - Global archiving of articles } \\ \text { - Immediate, unrestricted online access }\end{array}$

